\title{
Electroproduction of vector mesons
}

\section{Aharon Levy \\ Tel Aviv University}

on behalf the ZEUS collaboration 


\section{outline}

\section{Soft - hard}

$$
\boldsymbol{r}_{V} \equiv \frac{\sigma\left(\gamma^{*} \boldsymbol{p} \rightarrow \boldsymbol{V p}\right)}{\sigma_{t o t}\left(\gamma^{*} \boldsymbol{p}\right)}
$$

\section{Effective scale}




\section{soft - hard}

Energy dependence of cross section $\Rightarrow$ dynamics of process.

W dependence Regge type $(D L) \Rightarrow$ process called soft.

Example for soft: total hadron-p cross section.

$W$ dependence described by $P Q C D \Rightarrow$ process called hard.

Example for hard: Exclusive J/ $\psi$ electroproduction.

At HERA see interplay of soft and hard. 


\section{soft - hard transition}
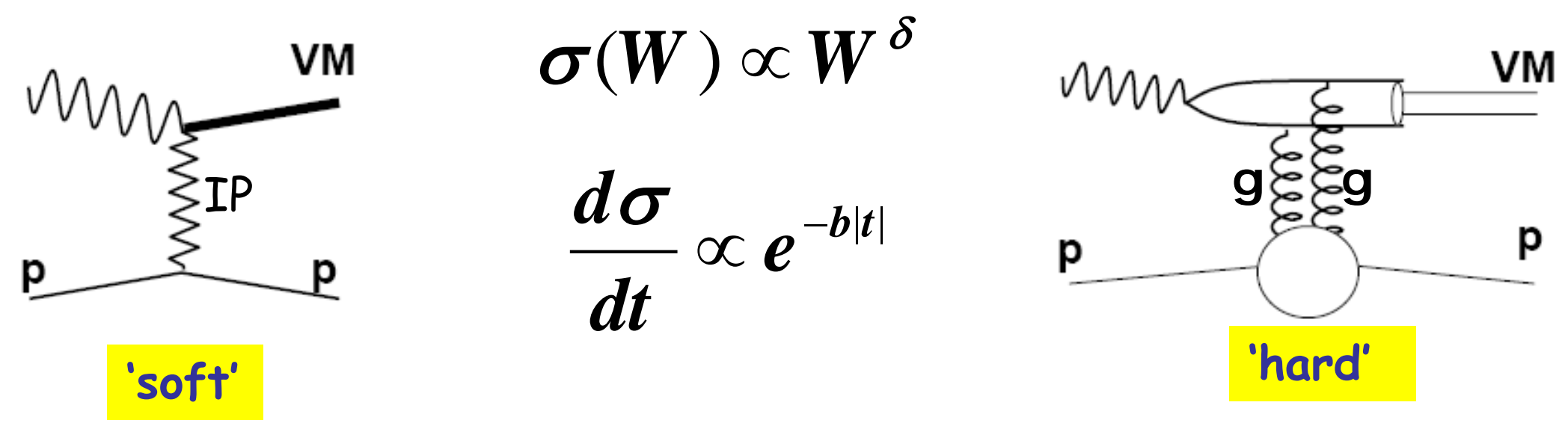

- Expect $\delta$ to increase from soft ( 0.2, from 'soft Pomeron' value) to hard $\left(\sim 0.8\right.$, from $\left.x g\left(x, Q^{2}\right)^{2}\right)$

- Expect $b$ to decrease from soft $\left(\sim 10 \mathrm{GeV}^{-2}\right)$ to hard ( 4-5 GeV-2) 


\section{Upsilon photoproduction}

\section{ZEUS}
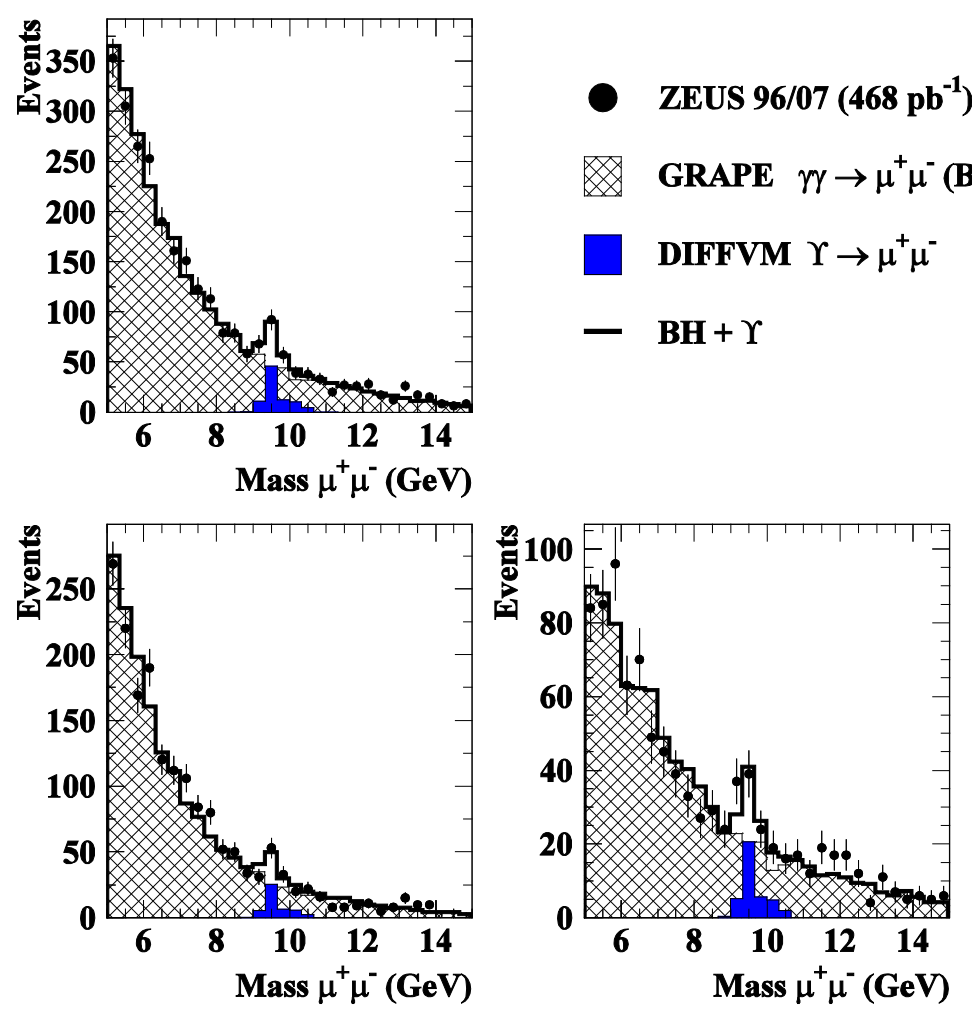

ZEUS

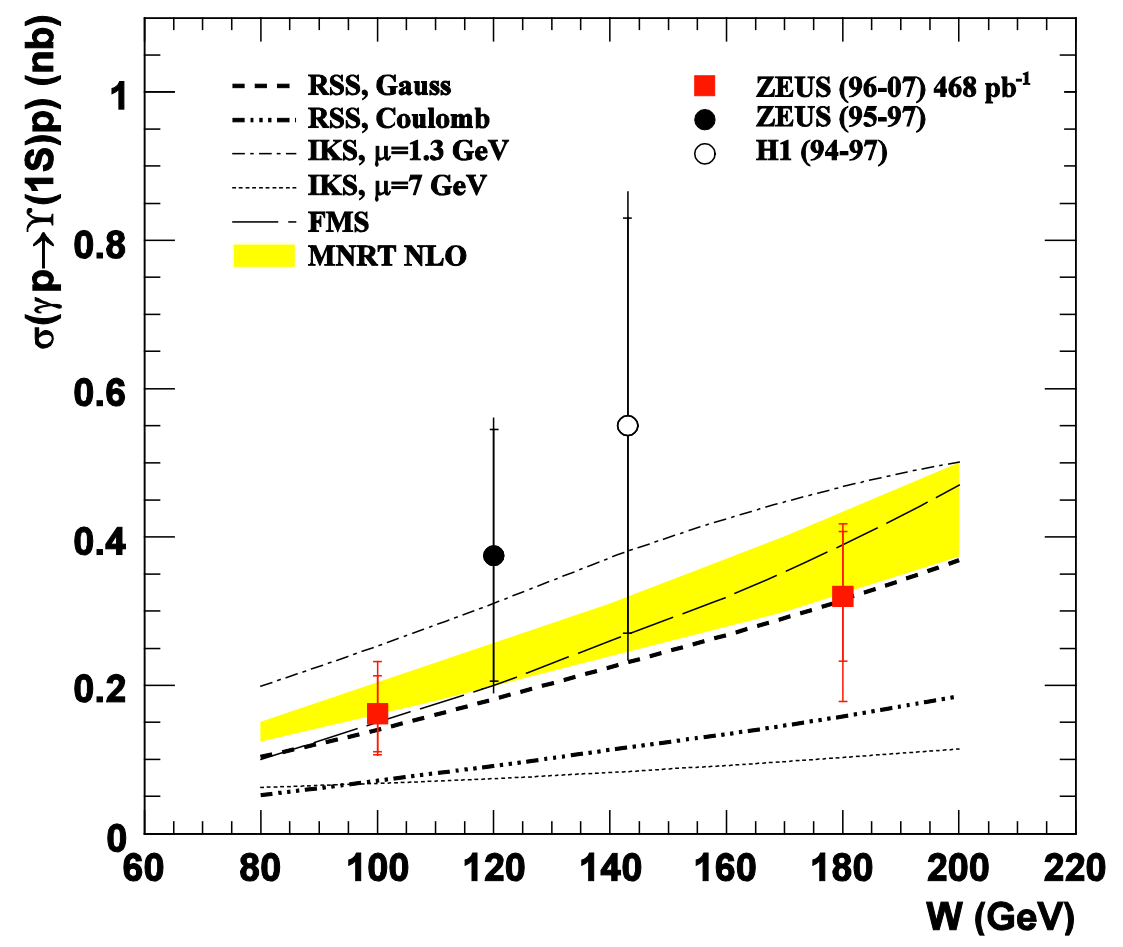




\section{$\sigma(W)-\rho^{0}$}

Fix mass - increase photon scale

\section{ZEUS}

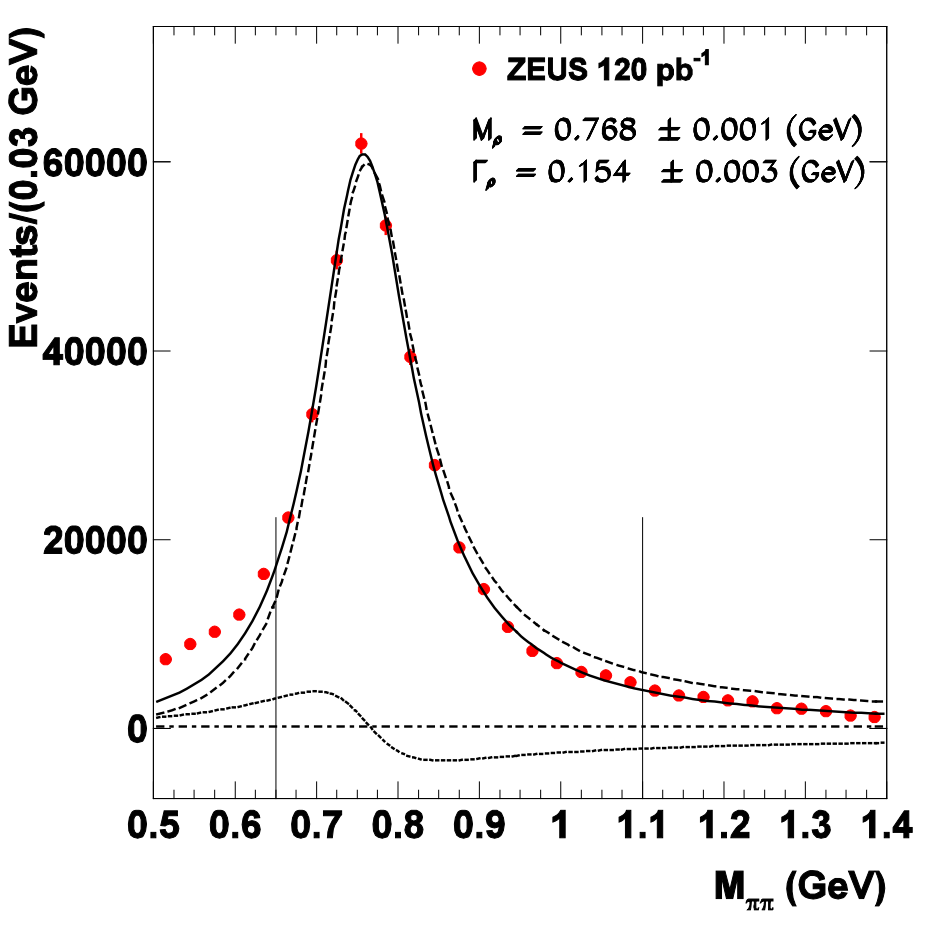

\section{ZEUS}
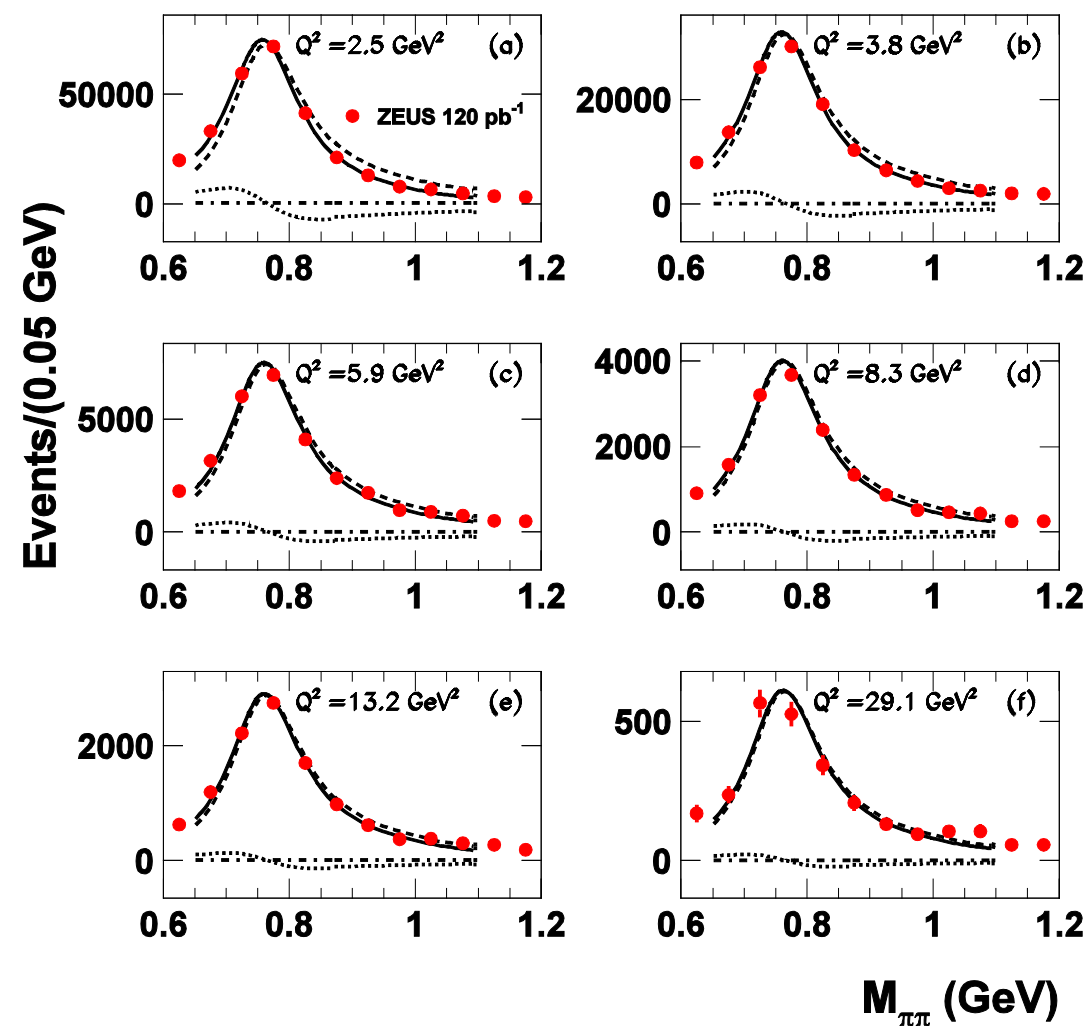


\section{$\sigma(W)-\rho^{0}$}

Fix mass - increase photon scale

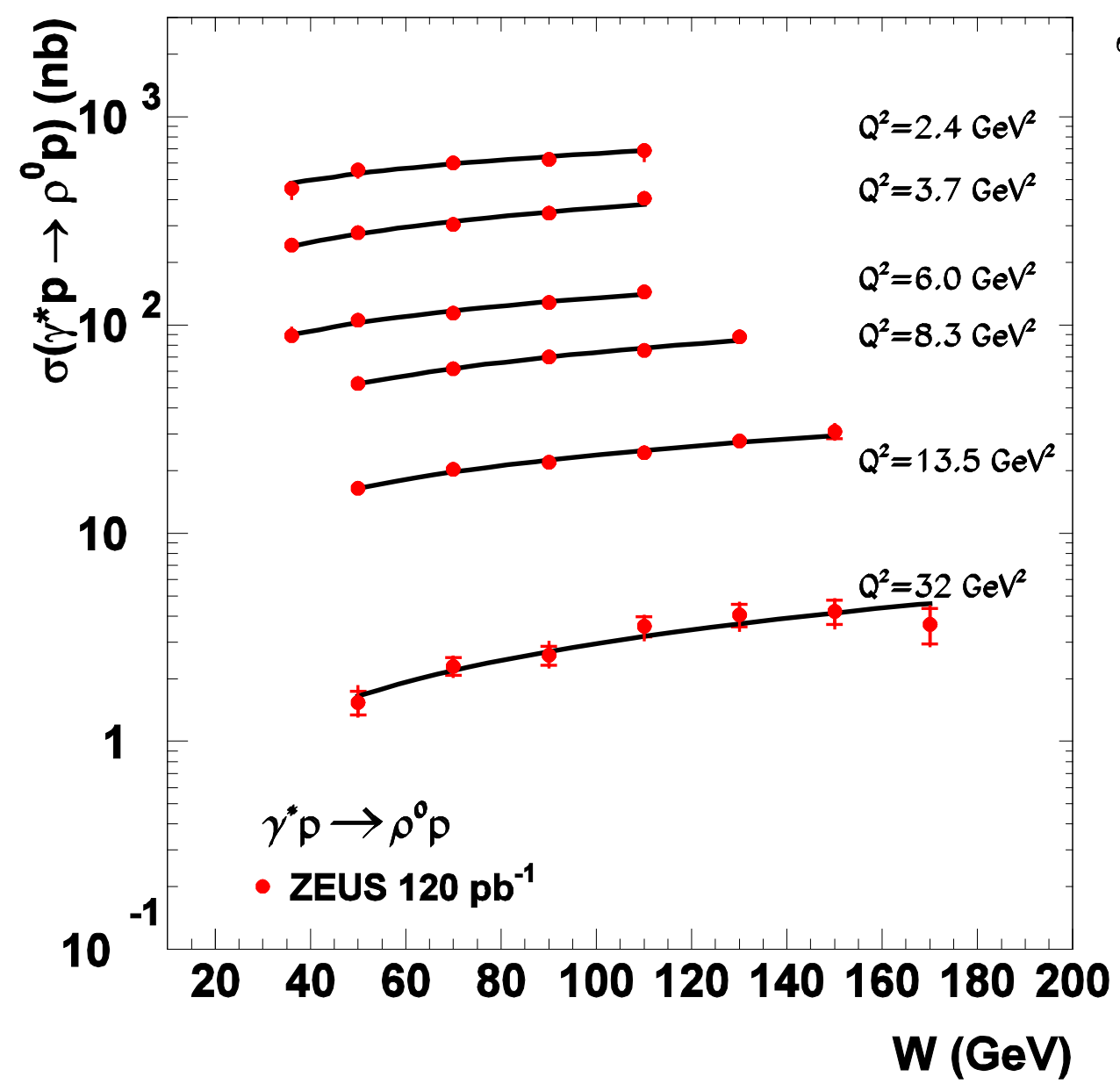

\section{$\sigma \propto W^{\delta}$}

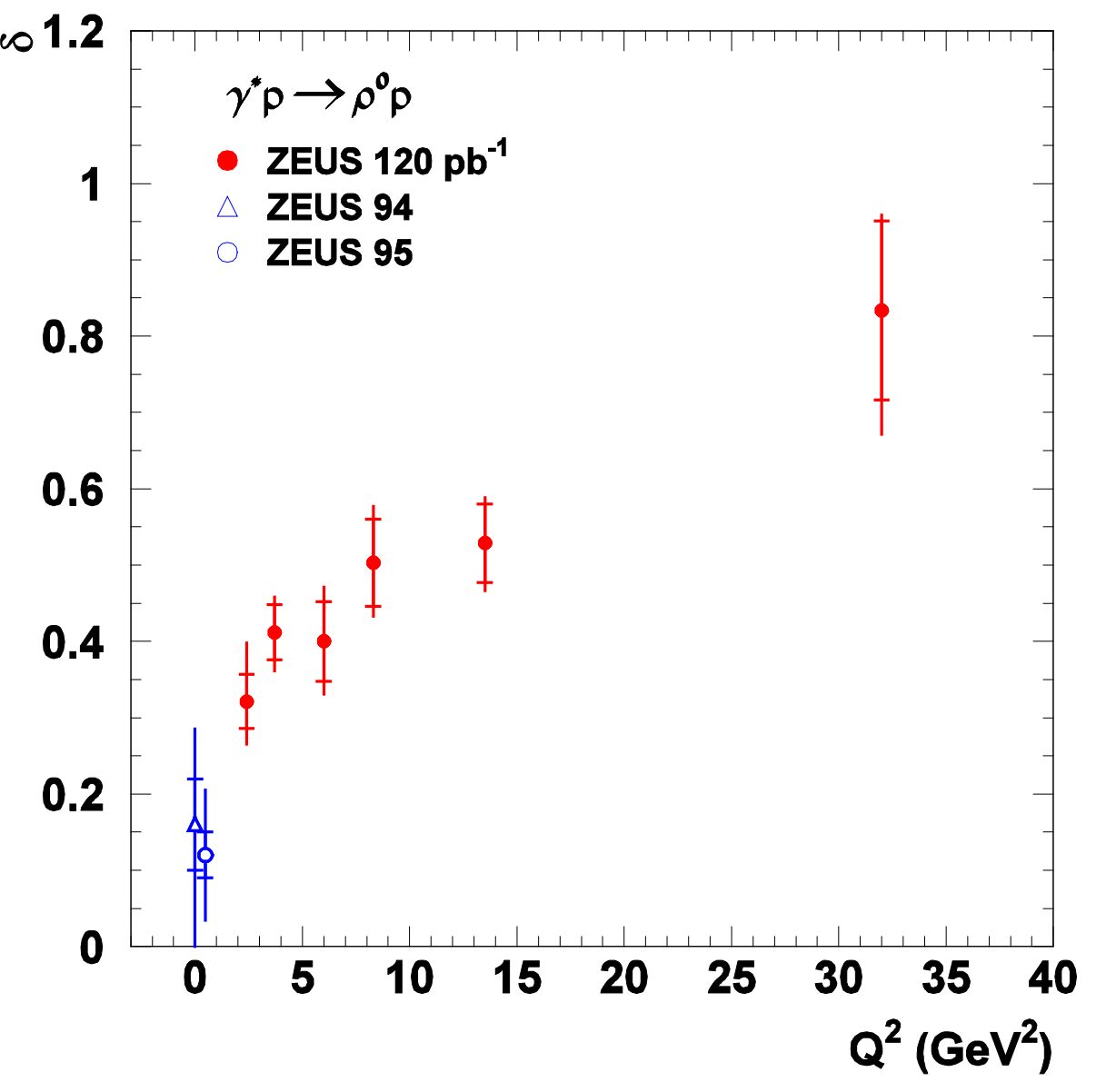

April 28, 2009

A. Levy: Exclusive VM, DIS09, 


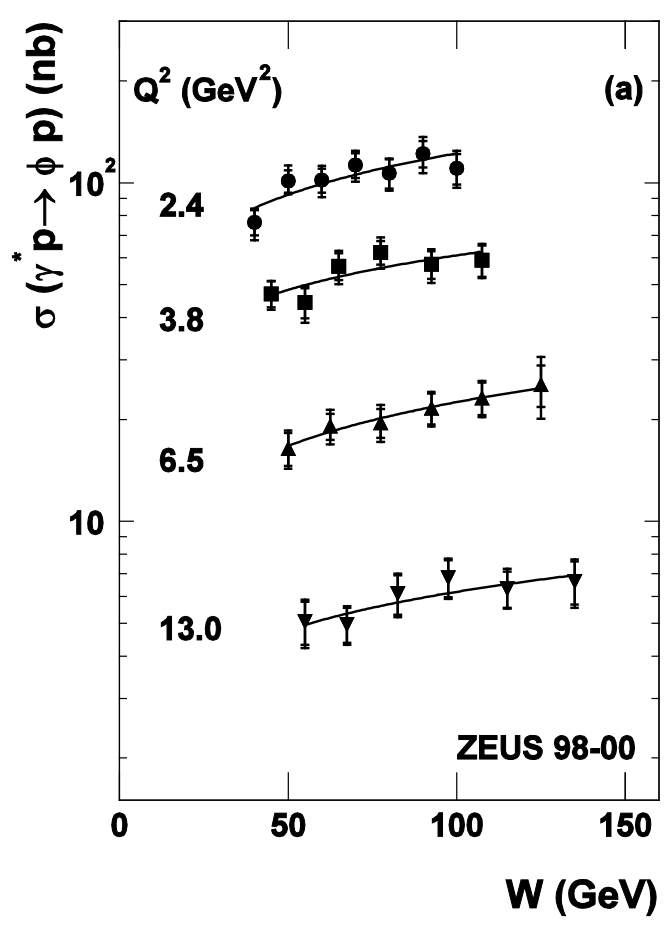

$\sigma(\mathrm{W})-\phi, J / \psi, \gamma$

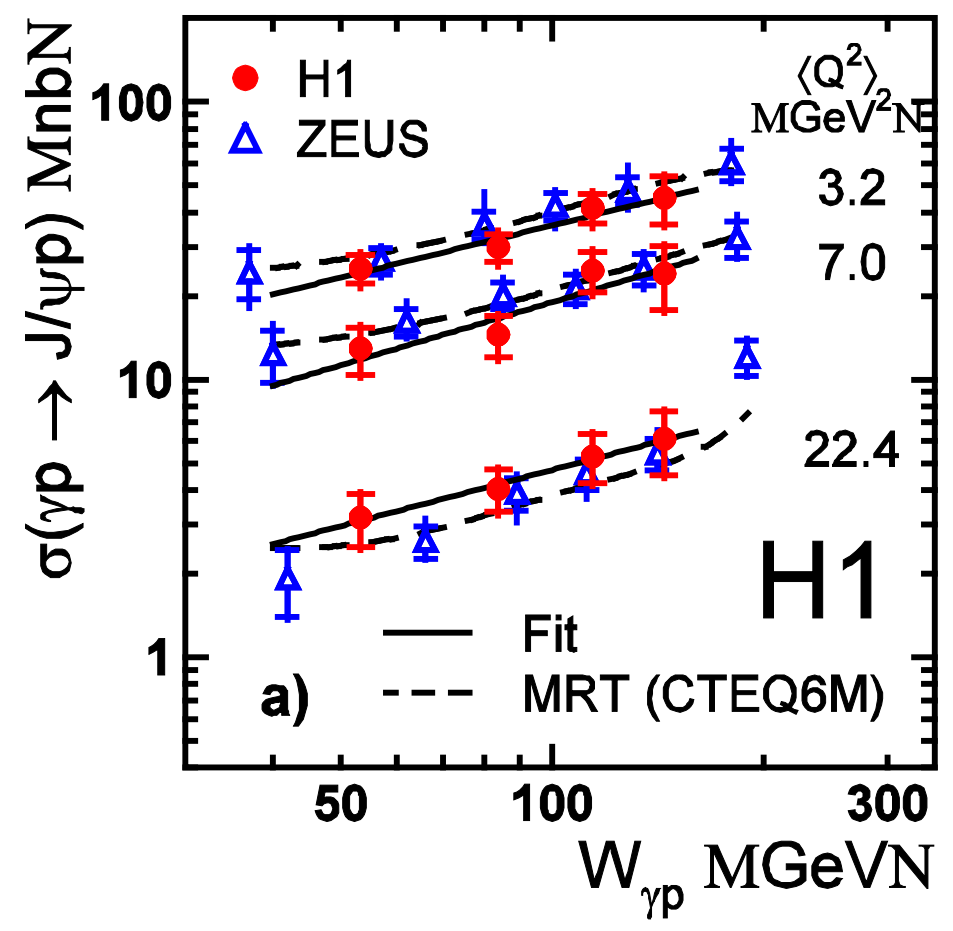

April 28, 2009

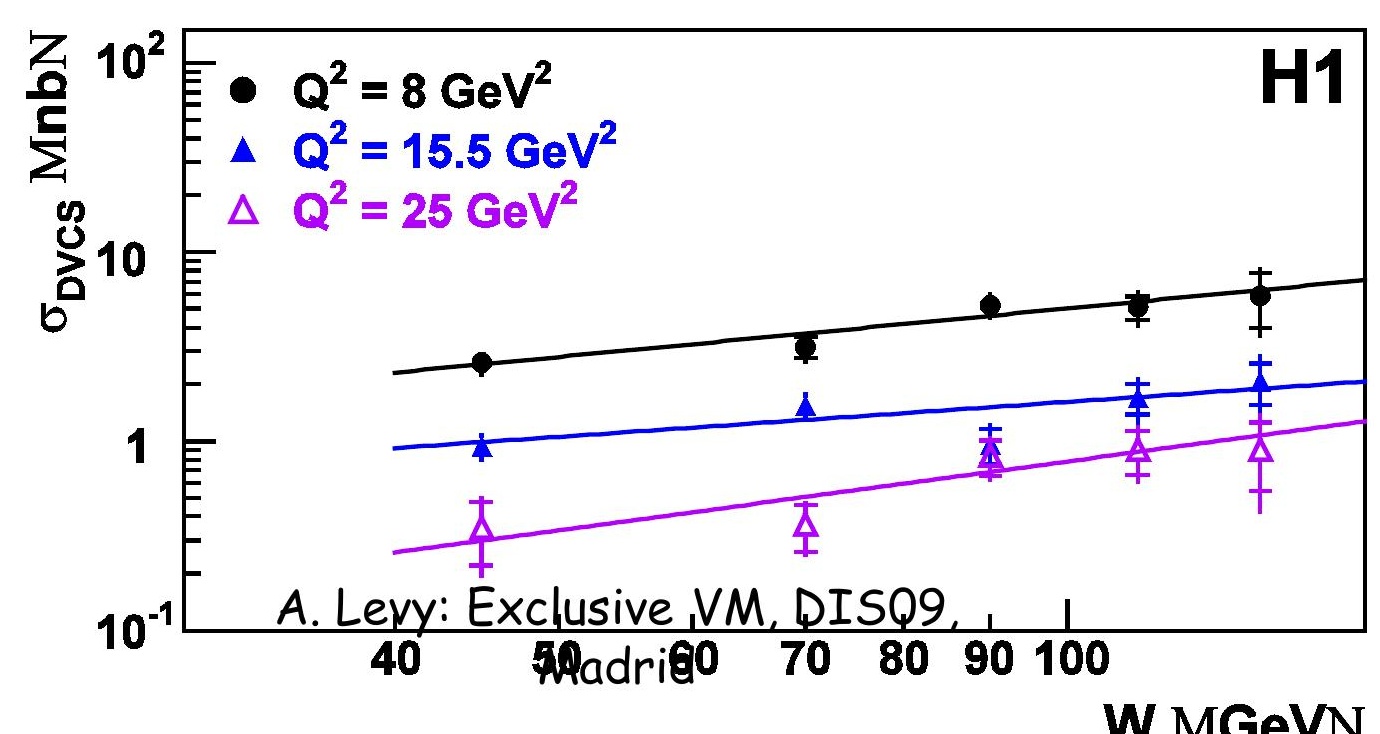




\section{DVCS - ZEUS}
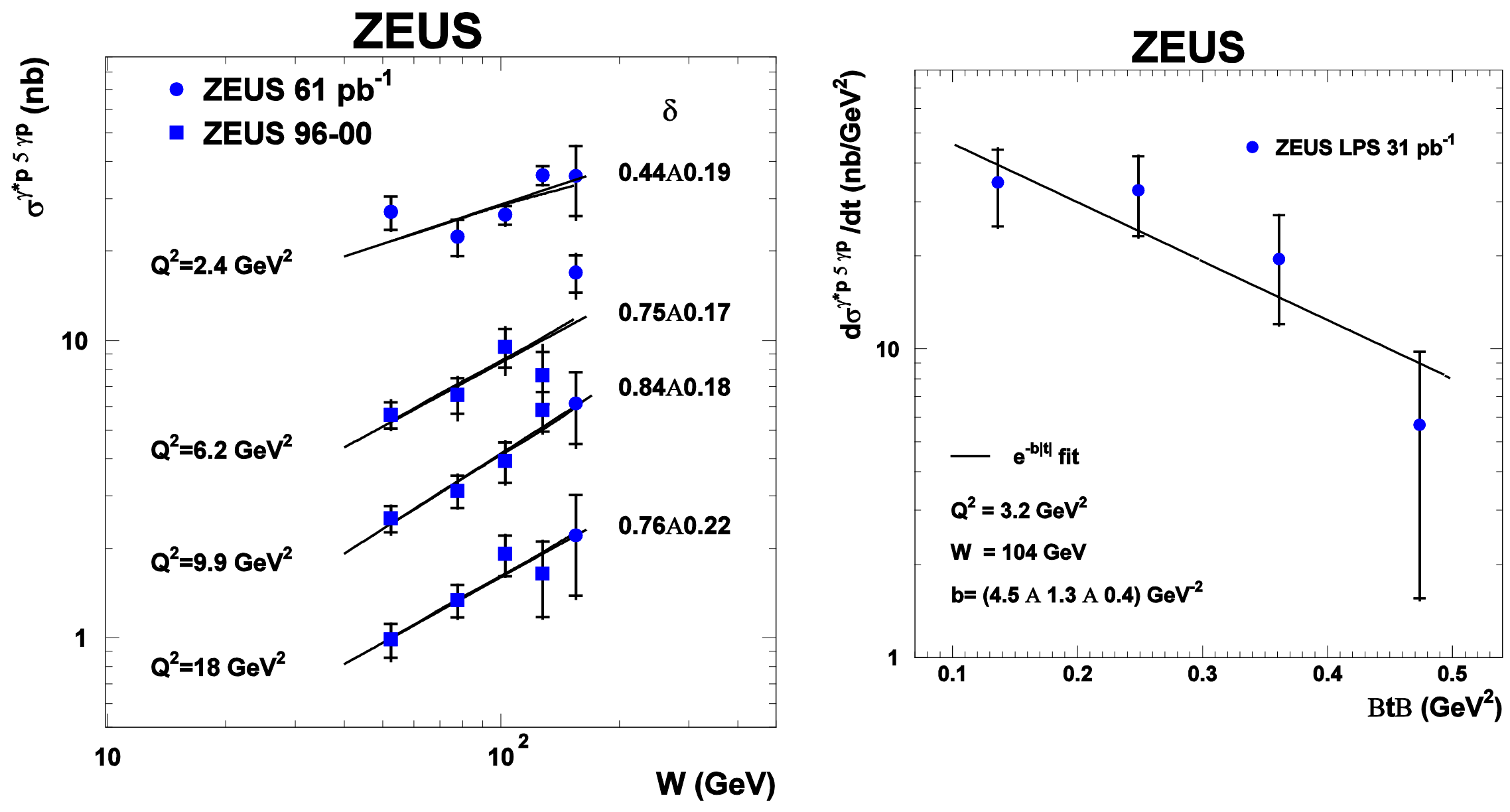

April 2009,28

A. Levy: Exclusive VM, DIS09, Madrid 


\section{$\delta\left(Q^{2}+M^{2}\right)-V M$}

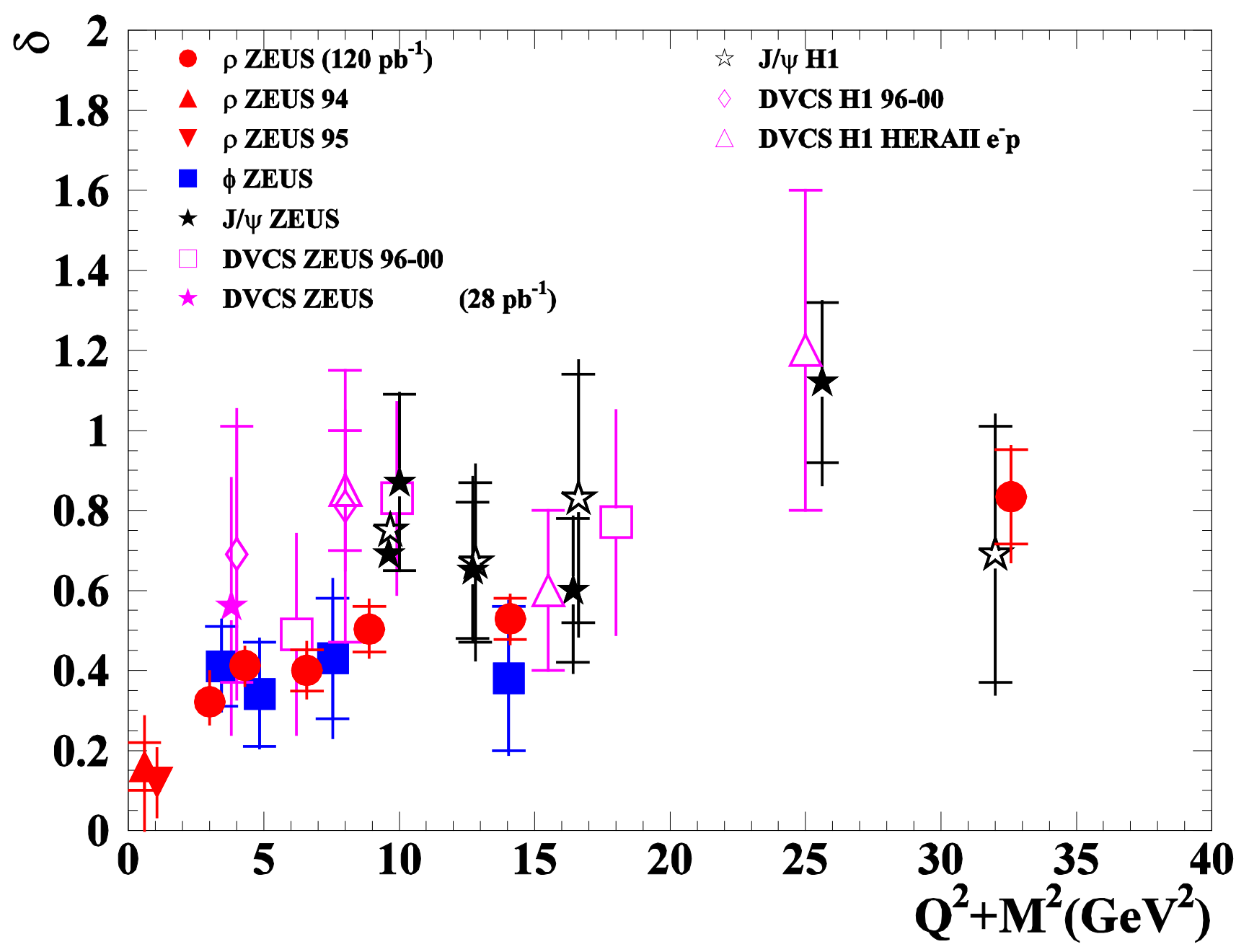

$W$ dependence becomes steeper as scale increases 


\section{$b\left(Q^{2}+M^{2}\right)-V M$}

\section{ZEUS}

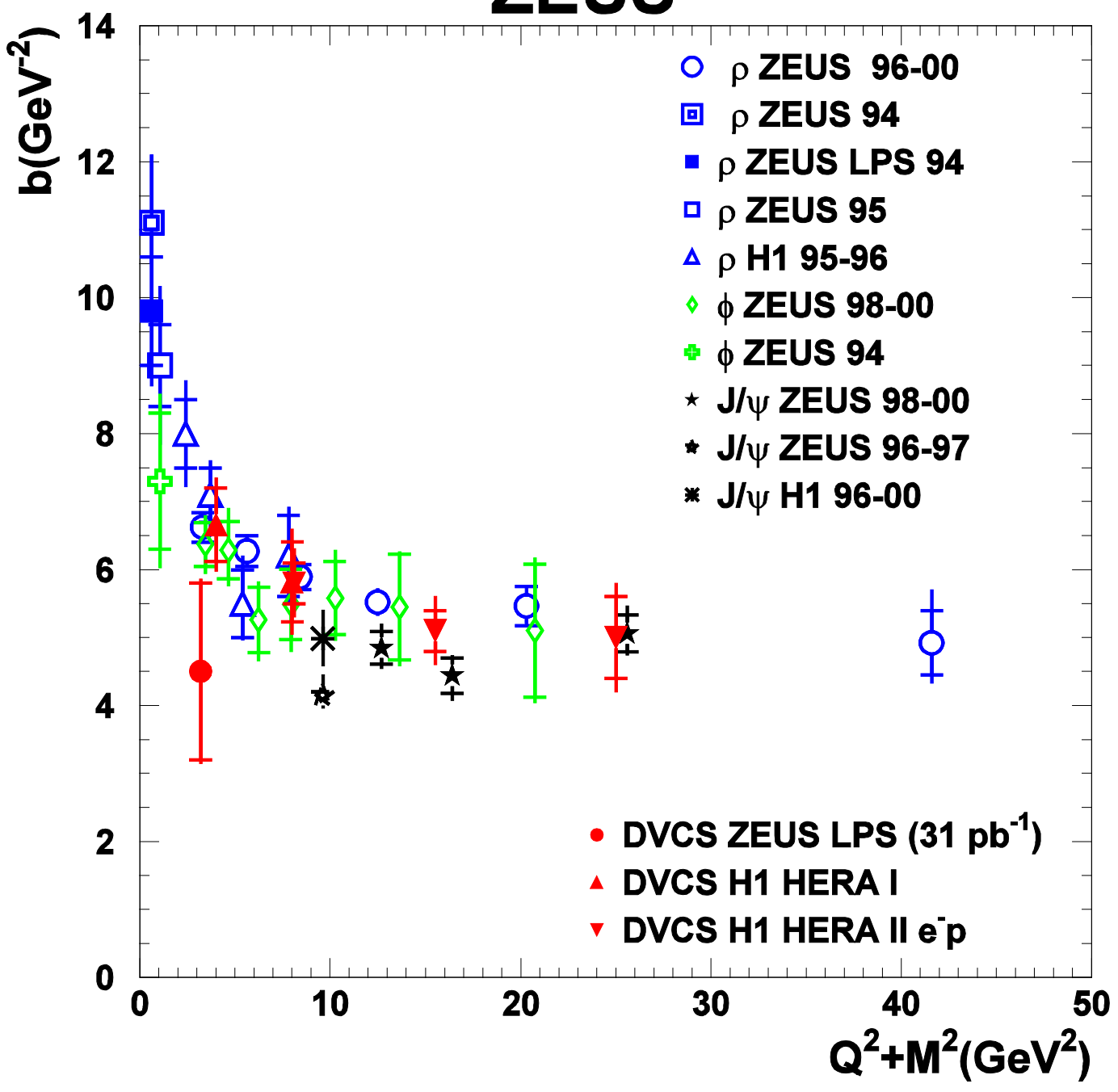

April 28, 2009

A. Levy: Exclusive VM, DIS09, Madrid 


\section{Ratio of diff/tot $\left(Q^{2}\right)$}

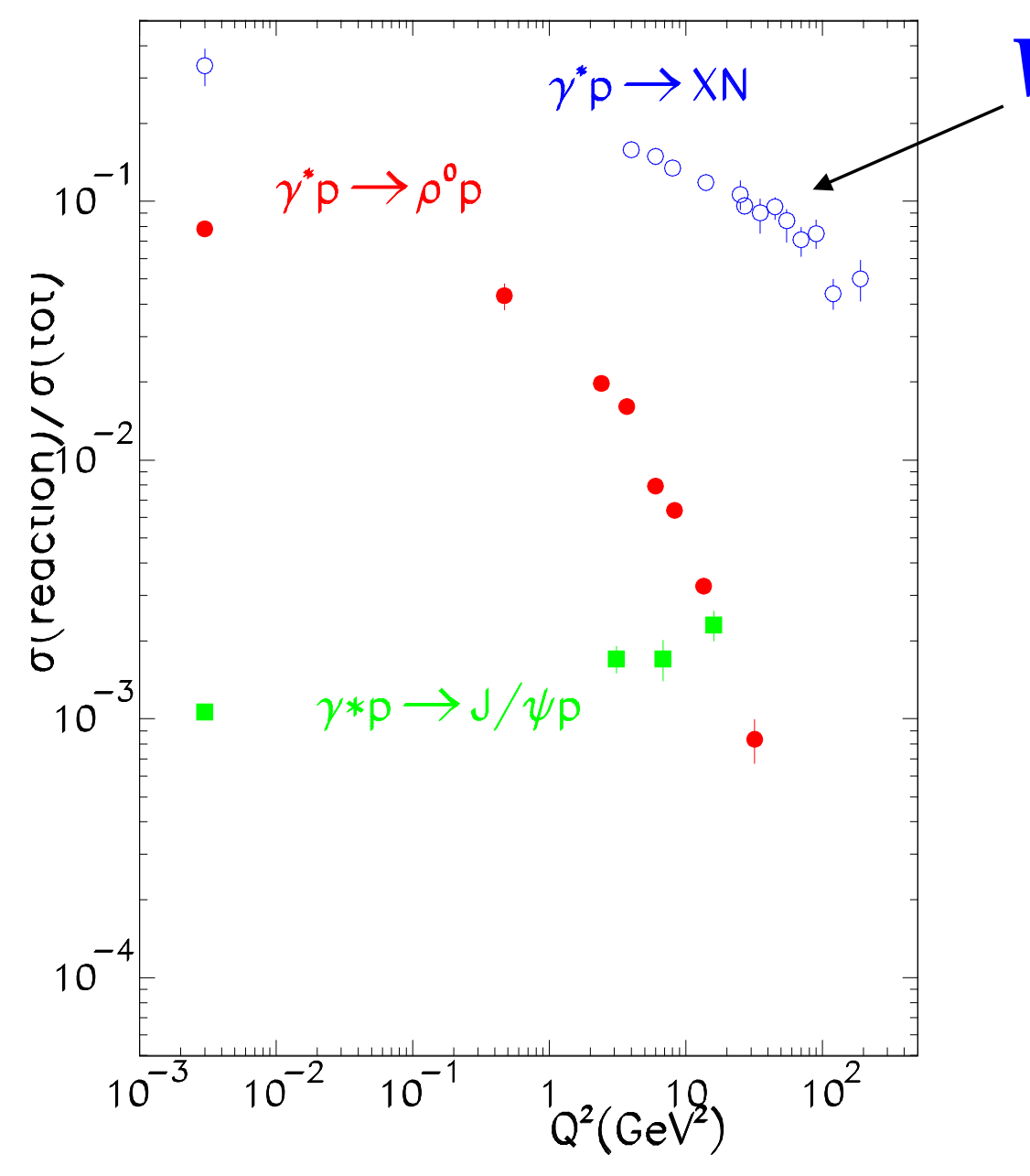

$W=220 \mathrm{GeV}, 0.28<\mathrm{M}_{X}<35 \mathrm{GeV}$

Decrease artificial; as $Q^{2}$ increases, $x_{I P}$ range decreases. Better to look at $F_{2} D / F_{2}$ as function of $Q^{2}$ at fixed $x_{I P}, \beta$ :

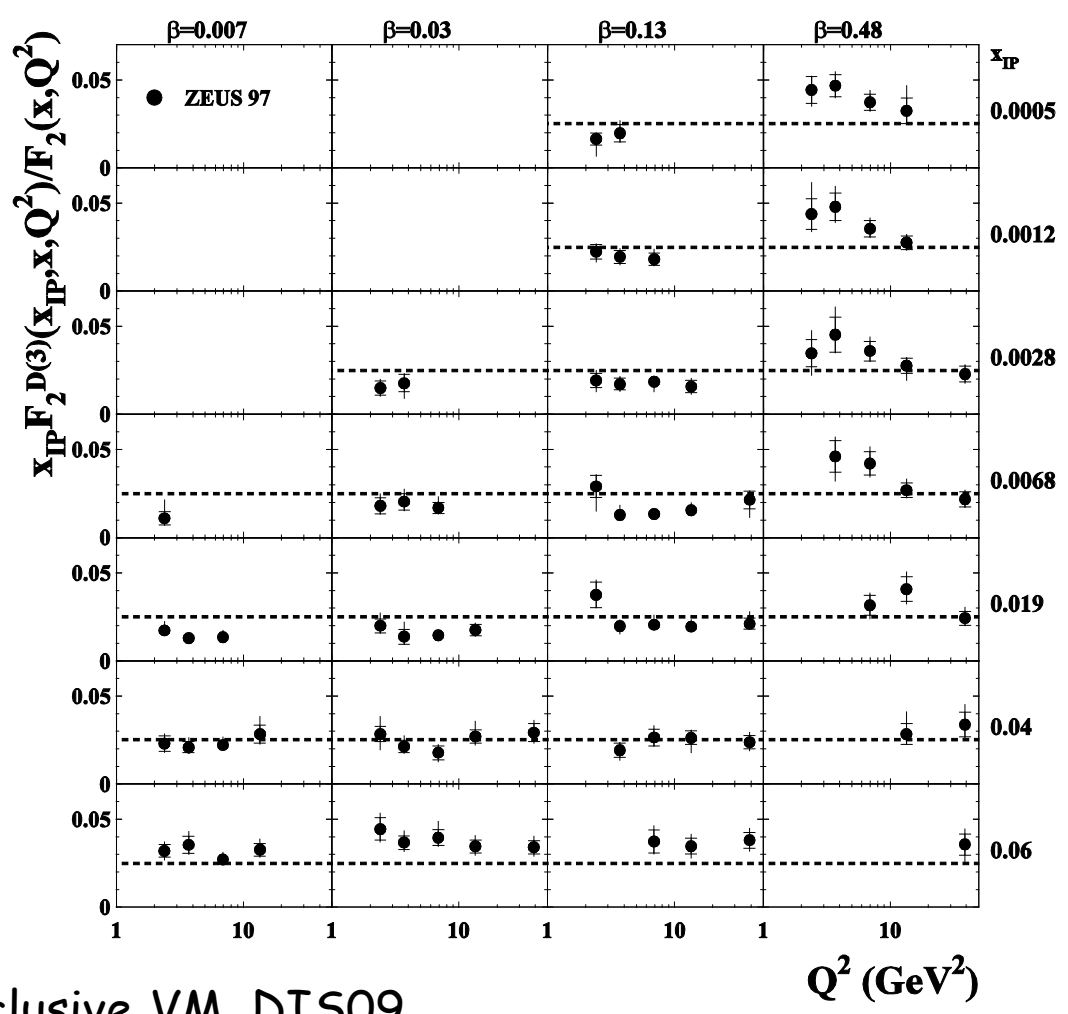

April 28, 2009

A. Levy: Exclusive VM, DIS09, Madrid 


\section{Ratio of VM/tot (W)}

$$
\boldsymbol{r}_{V} \equiv \frac{\sigma\left(\gamma^{*} \boldsymbol{p} \rightarrow \boldsymbol{V} \boldsymbol{p}\right)}{\sigma_{\text {tot }}\left(\gamma^{*} \boldsymbol{p}\right)} \quad \boldsymbol{F}_{2} \propto \boldsymbol{x}^{-\lambda}
$$

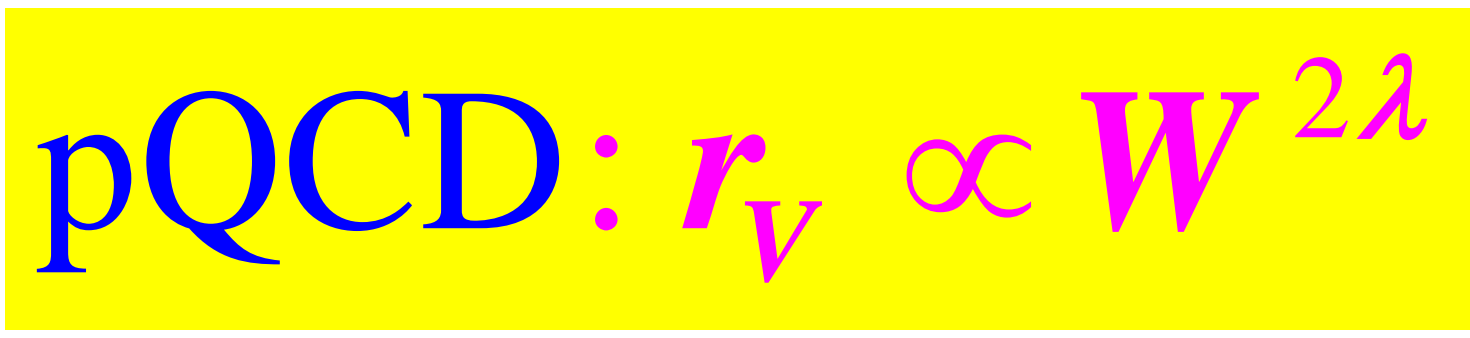

$$
\begin{aligned}
& \text { Regge: } r_{V} \propto W^{2 \lambda} \\
& \left(\lambda \equiv \alpha_{I P}(0)-1\right)
\end{aligned}
$$




\section{$\sigma\left(\gamma^{\star} V \rightarrow V p\right) / \sigma_{\text {tot }}\left(\gamma^{*} p\right)-p Q C D$}

$\left.\frac{d \sigma_{L}}{d t}\right|_{t=0} \propto \frac{1}{Q^{6}} \alpha_{S}^{2}\left(Q^{2}\right)\left[x \boldsymbol{g}\left(x, Q^{2}\right)\right]^{2} \propto x^{-2 \lambda}$ for fixed $Q^{2}$ $\frac{d \sigma_{V}}{d t} \propto \boldsymbol{e}^{-b|t|}, \sigma_{t o t}\left(\gamma^{*} \boldsymbol{p}\right) \propto \frac{\boldsymbol{F}_{2}}{\mathbf{Q}^{2}} \propto \boldsymbol{x}^{-\lambda}$ for fixed $\mathrm{Q}^{2}$, low $\mathrm{x}$ $\boldsymbol{r}_{V} \equiv \frac{\boldsymbol{\sigma ( \gamma ^ { * } \boldsymbol { p } \rightarrow \boldsymbol { V } \boldsymbol { p } )}}{\sigma_{\text {tot }}\left(\gamma^{*} \boldsymbol{p}\right)} \propto\left(1+\frac{1}{\boldsymbol{R}}\right) \frac{\boldsymbol{x}^{-\lambda}}{\boldsymbol{b}} \propto \frac{\boldsymbol{x}^{-\lambda}}{\boldsymbol{b}}=\frac{W^{2 \lambda}}{\boldsymbol{b}}$ for fixed $\mathrm{Q}^{2}$

$R$ is $W$ independent (for fixed $Q^{2}$ ): $a^{\prime}$ small $\Rightarrow b$ slow $W$ dependence 


\section{Effective Pomeron trajectory}

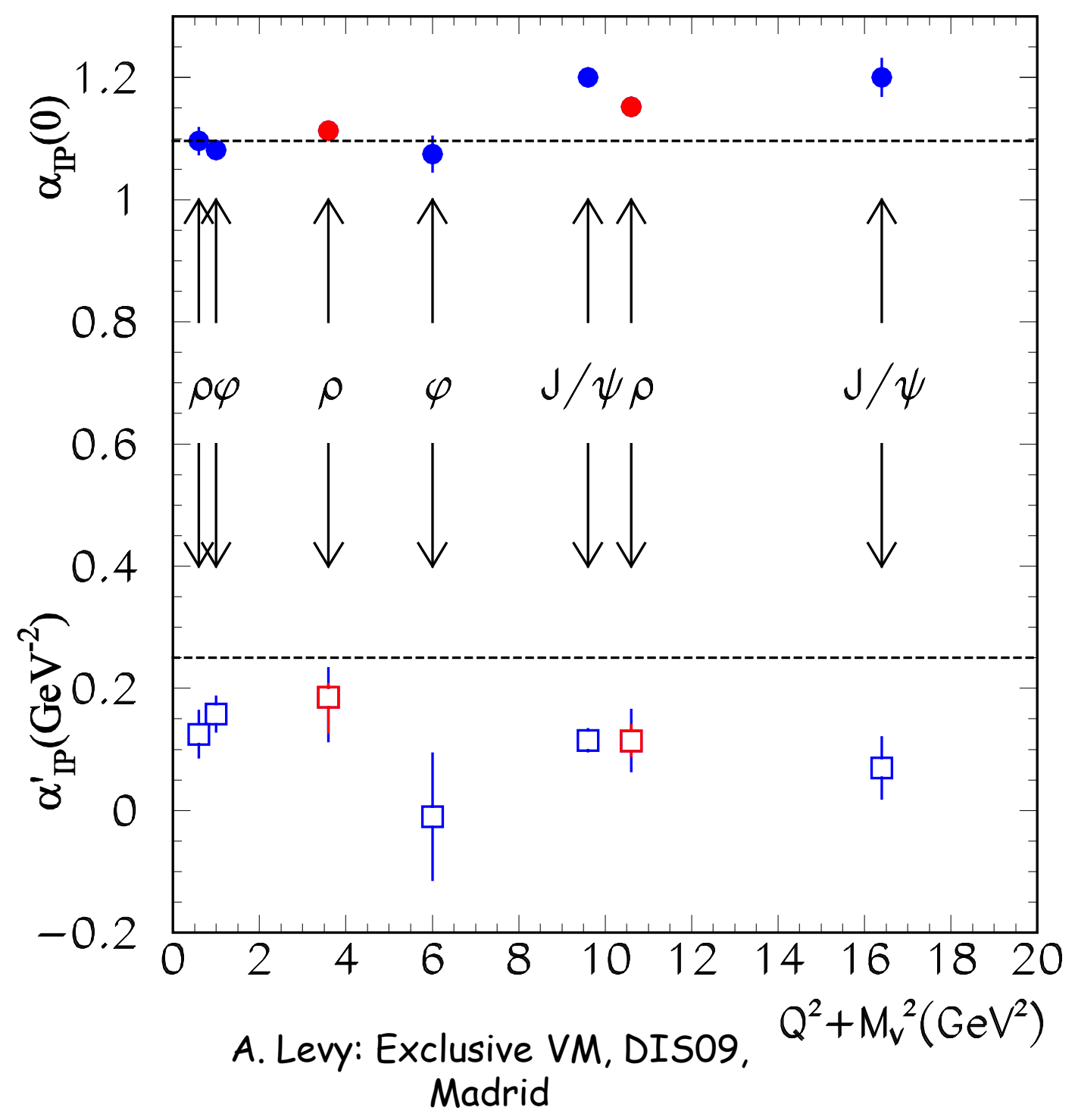




\section{$\sigma\left(\gamma^{\star} V \rightarrow V p\right) / \sigma_{\text {tot }}\left(\gamma^{\star} p\right)-$ Regge}

$$
\begin{gathered}
\boldsymbol{\sigma}_{\text {tot }}\left(\boldsymbol{\gamma}^{*} \boldsymbol{p}\right) \propto \boldsymbol{W}^{2\left(\alpha_{\boldsymbol{P}}(0)-1\right)} \\
\boldsymbol{\sigma}\left(\gamma^{*} \boldsymbol{p} \rightarrow \boldsymbol{V p}\right) \propto \frac{\boldsymbol{W}^{4\left(\alpha_{P}(0)-1\right)}}{\boldsymbol{b}} \\
\boldsymbol{r}_{\boldsymbol{V}} \propto \frac{\boldsymbol{W}^{2\left(\alpha_{P}(0)-1\right)}}{\boldsymbol{b}}
\end{gathered}
$$

$\alpha$ small $\Rightarrow b$ slow $W$ dependence

$\lambda \equiv \alpha_{p}(0)-1$ 


$$
\boldsymbol{F}_{2} \sim \boldsymbol{X}^{-\lambda\left(\boldsymbol{Q}^{2}\right)}
$$

$\sigma \propto \mathbf{S}^{0.096}$

\section{$\lambda$ HERA}

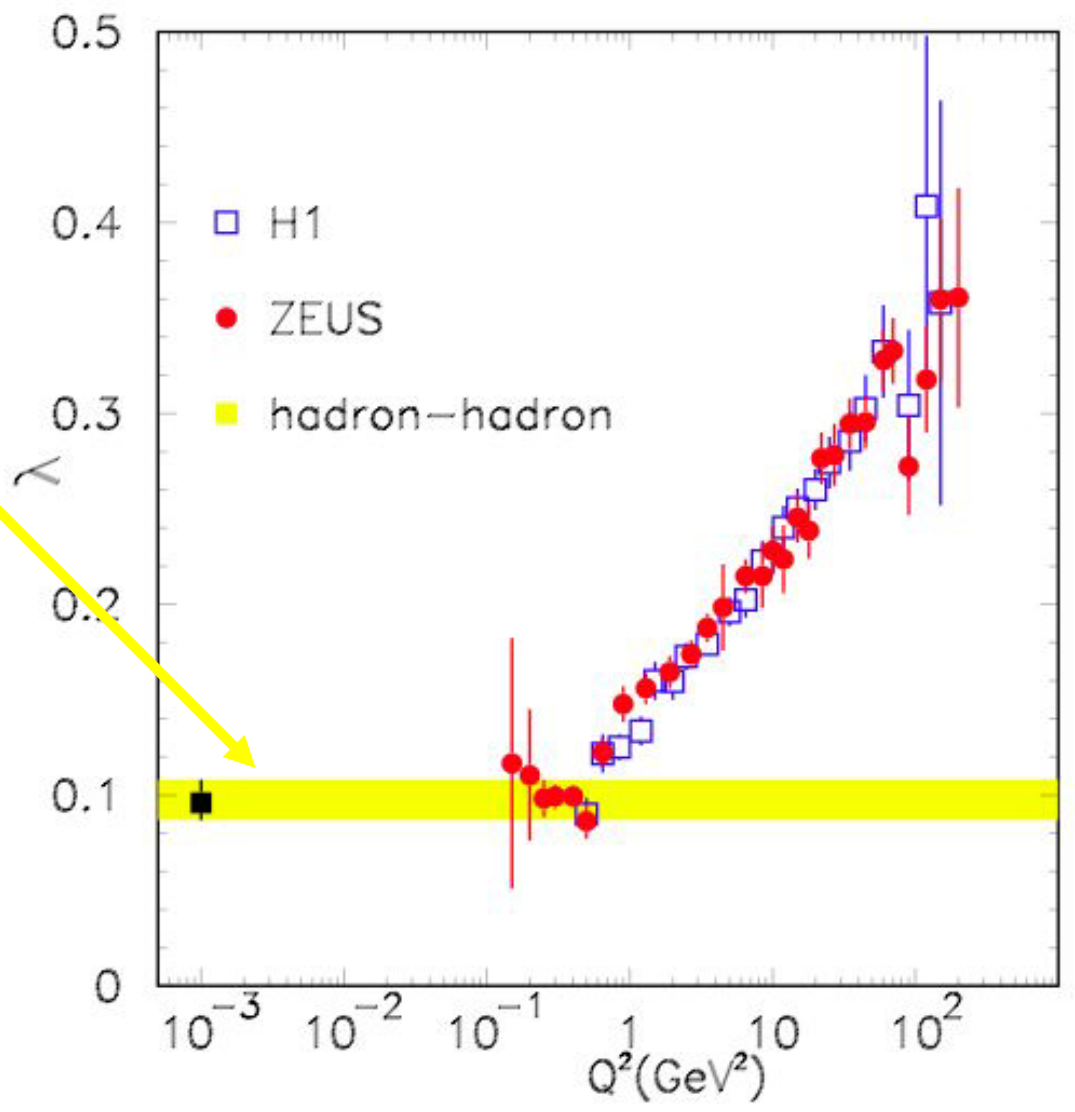

April 28, 2009

A. Levy: Exclusive VM, DIS09, 


\section{Ratio of VM/tot (W)}

$$
\boldsymbol{r}_{V} \equiv \frac{\sigma\left(\gamma^{*} \boldsymbol{p} \rightarrow \boldsymbol{V} \boldsymbol{p}\right)}{\sigma_{\text {tot }}\left(\gamma^{*} \boldsymbol{p}\right)} \quad \boldsymbol{F}_{2} \propto \boldsymbol{x}^{-\lambda}
$$

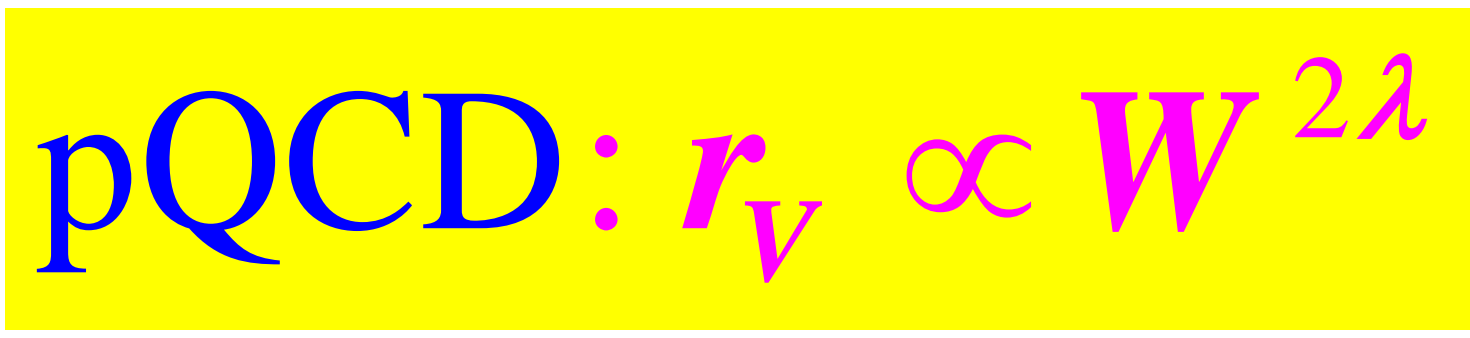

$$
\begin{aligned}
& \text { Regge: } r_{V} \propto W^{2 \lambda} \\
& \left(\lambda \equiv \alpha_{I P}(0)-1\right)
\end{aligned}
$$




\section{ratio - at what scale?}

Try the following:

$$
\begin{aligned}
& \boldsymbol{Q}_{\text {eff }}^{2}=\boldsymbol{Q}^{2} \\
& \boldsymbol{Q}_{\text {eff }}^{2}=\frac{\boldsymbol{Q}^{2}+\boldsymbol{M}_{\boldsymbol{V}}^{2}}{4} \\
& \boldsymbol{Q}_{\text {eff }}^{2}=\left(\frac{\boldsymbol{Q}^{2}}{2.65}\right)^{0.887} \\
& \boldsymbol{Q}_{\text {eff }}^{2}=0.23 \boldsymbol{e}^{\left(0.1 \mathbf{Q}^{2}\right)}
\end{aligned}
$$




\section{$\rho$}
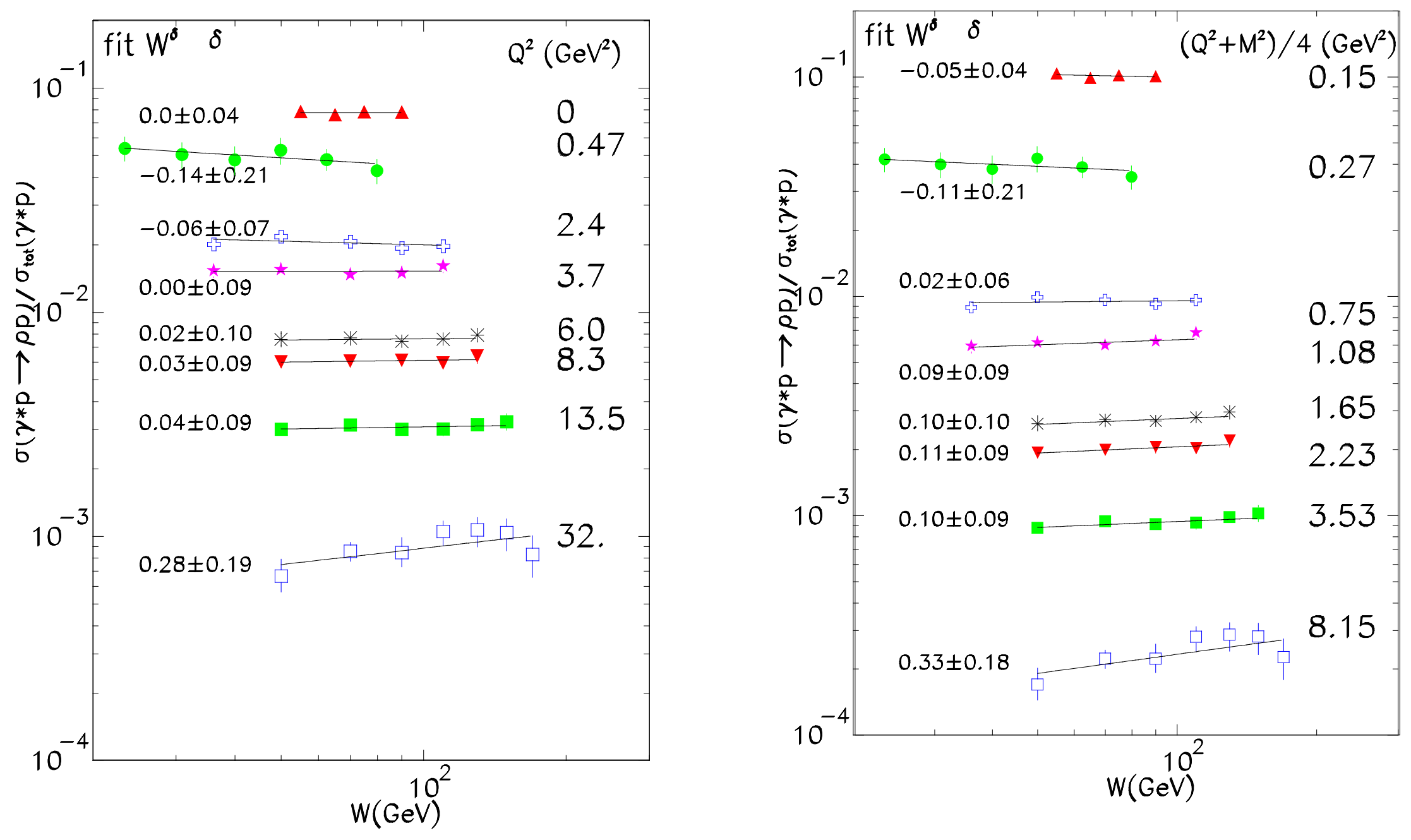

April 28, 2009

A. Levy: Exclusive VM, DIS09, 

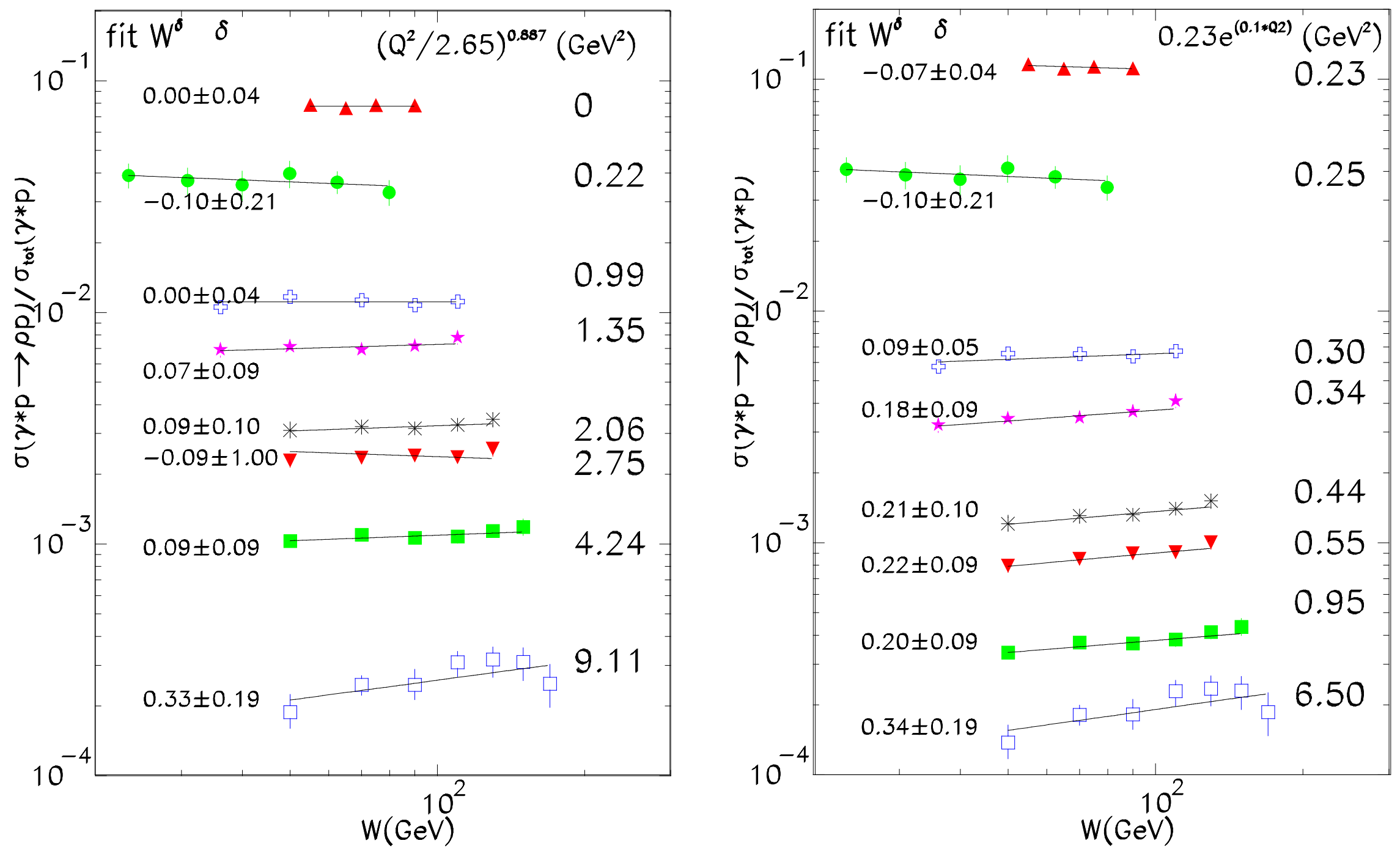

April 2009,28

A. Levy: Exclusive VM, DIS09,

23

Madrid 


\section{$\lambda$ plots $(\rho)$}

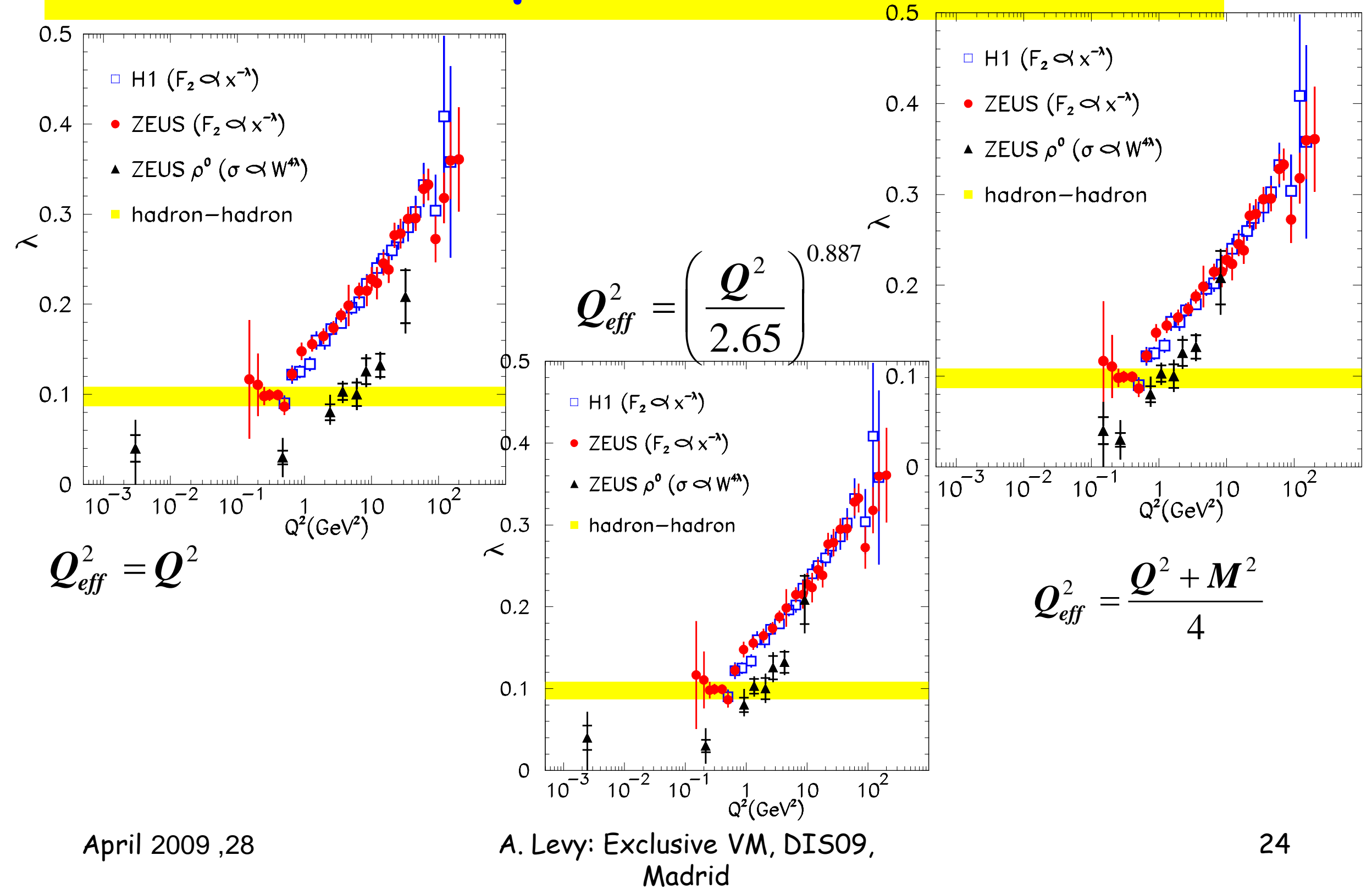




\section{$\lambda$ plots $(\rho)$}

$$
\boldsymbol{Q}_{\text {eff }}^{2}=0.23 \boldsymbol{e}^{0.1 Q^{2}}
$$

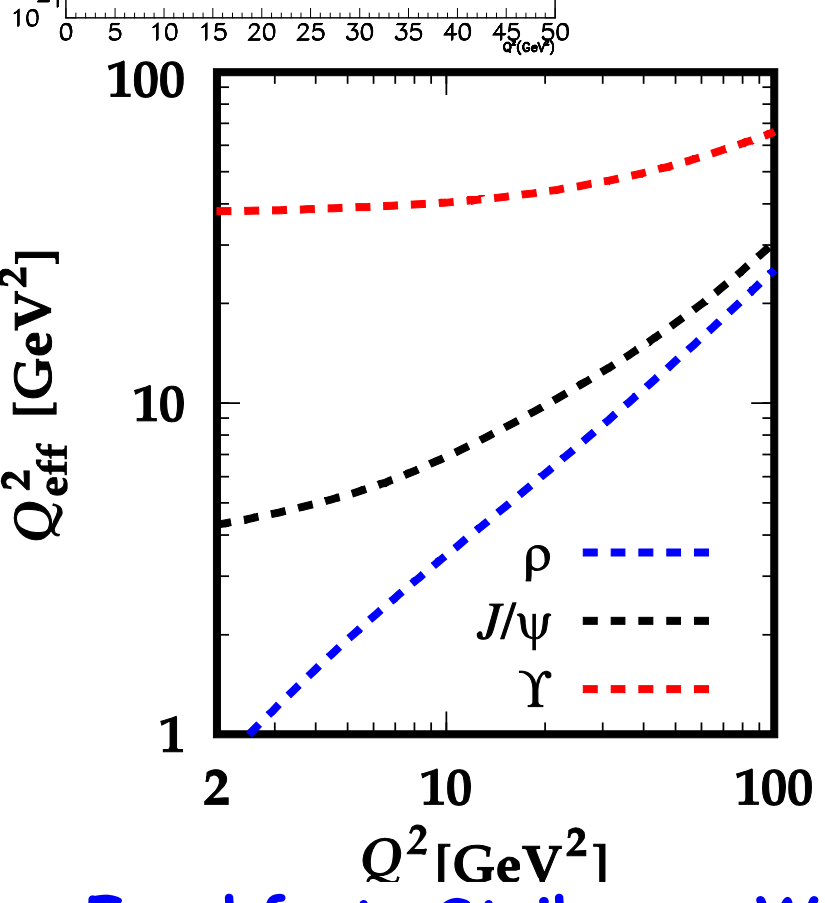

Frankfurt, Strikman, Weiss

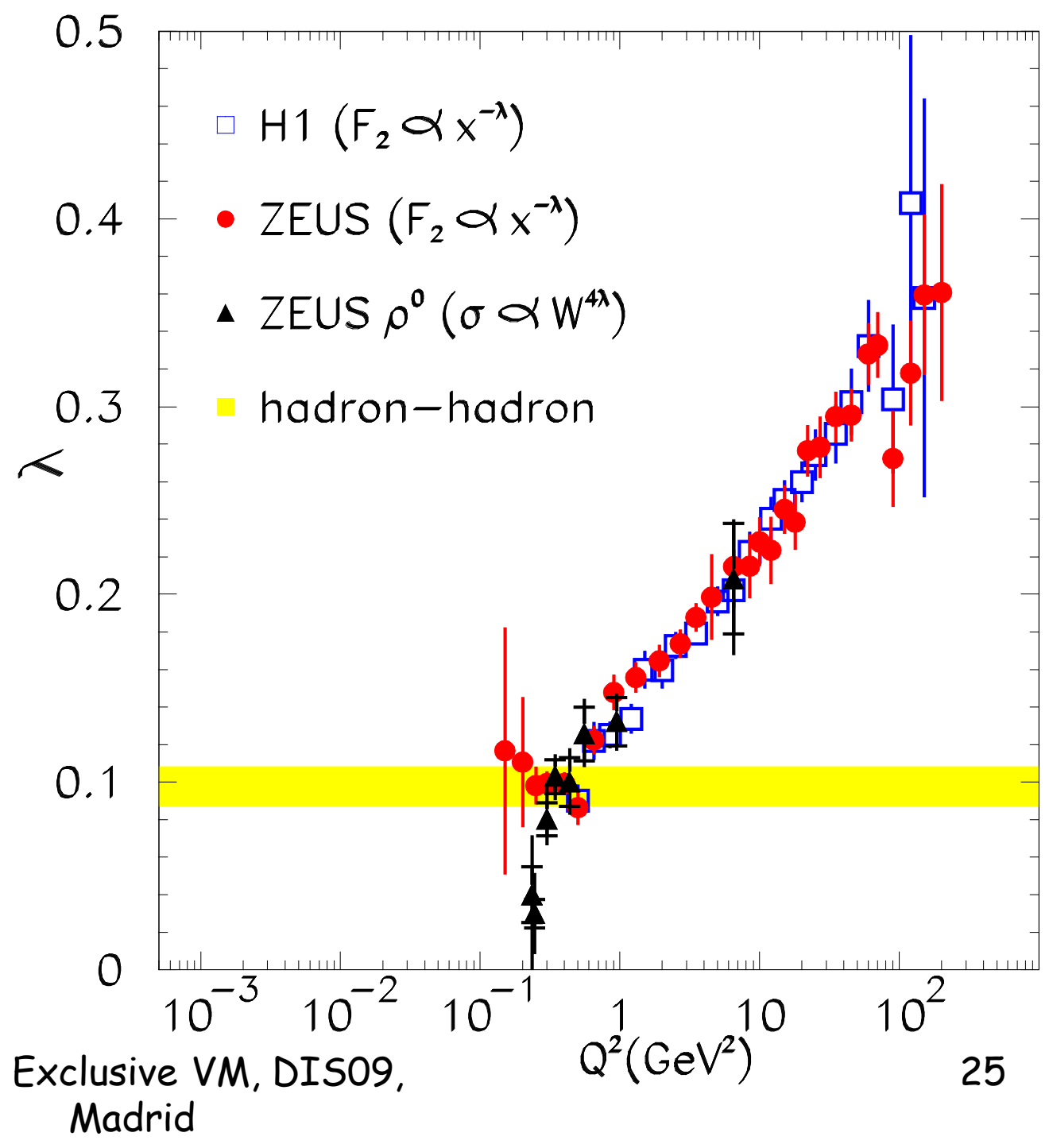




\section{$J / \psi$}
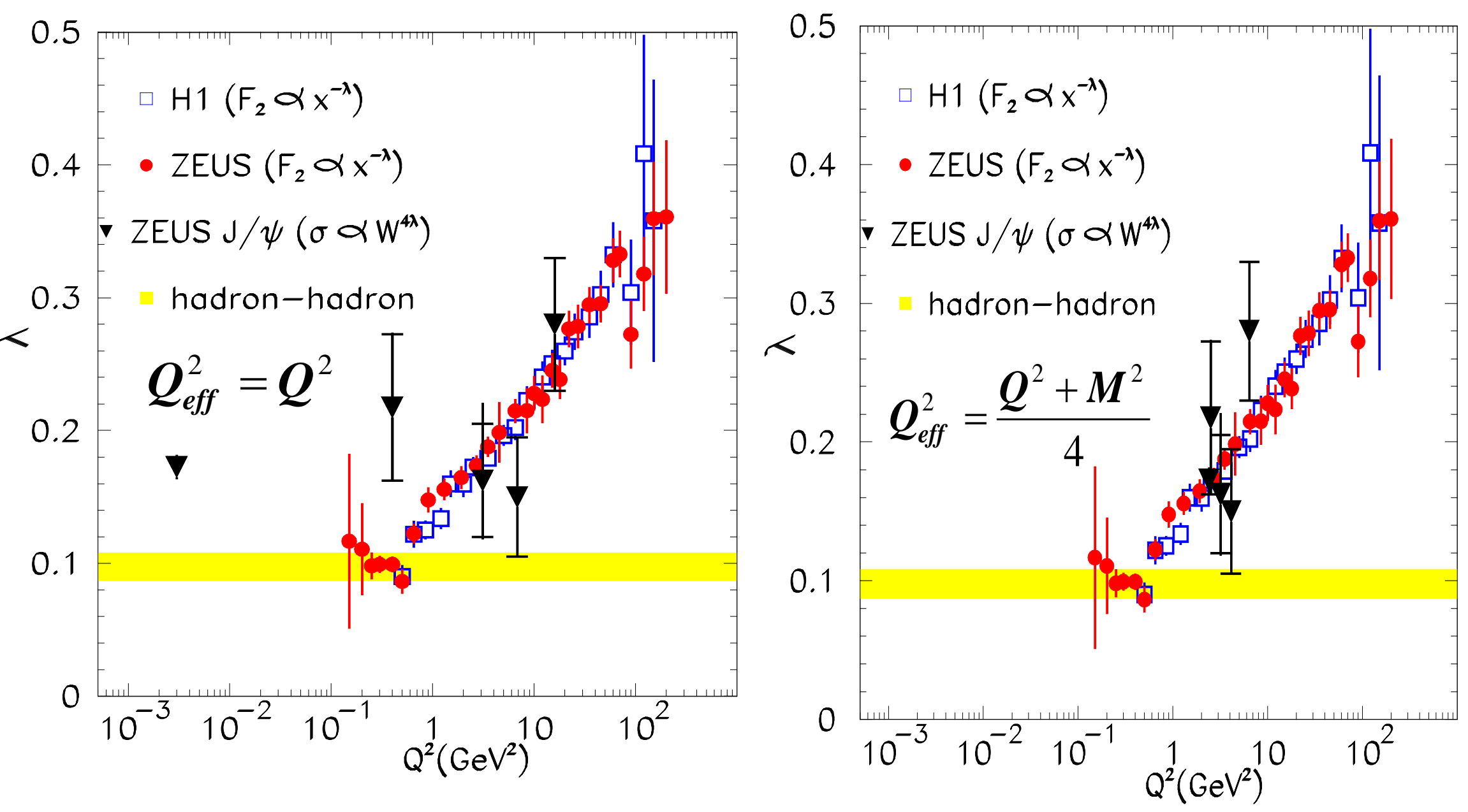

April 28, 2009

A. Levy: Exclusive VM, DIS09,

Madrid 


\section{Effective scale}

M.S.: $Q^{2}$ eff in the $\rho^{0}$ production case is much smaller than $Q^{2}$ of the photon due to presence of the convolution of the soft $\rho^{0}$ wave function and small size longitudinal photon wave function
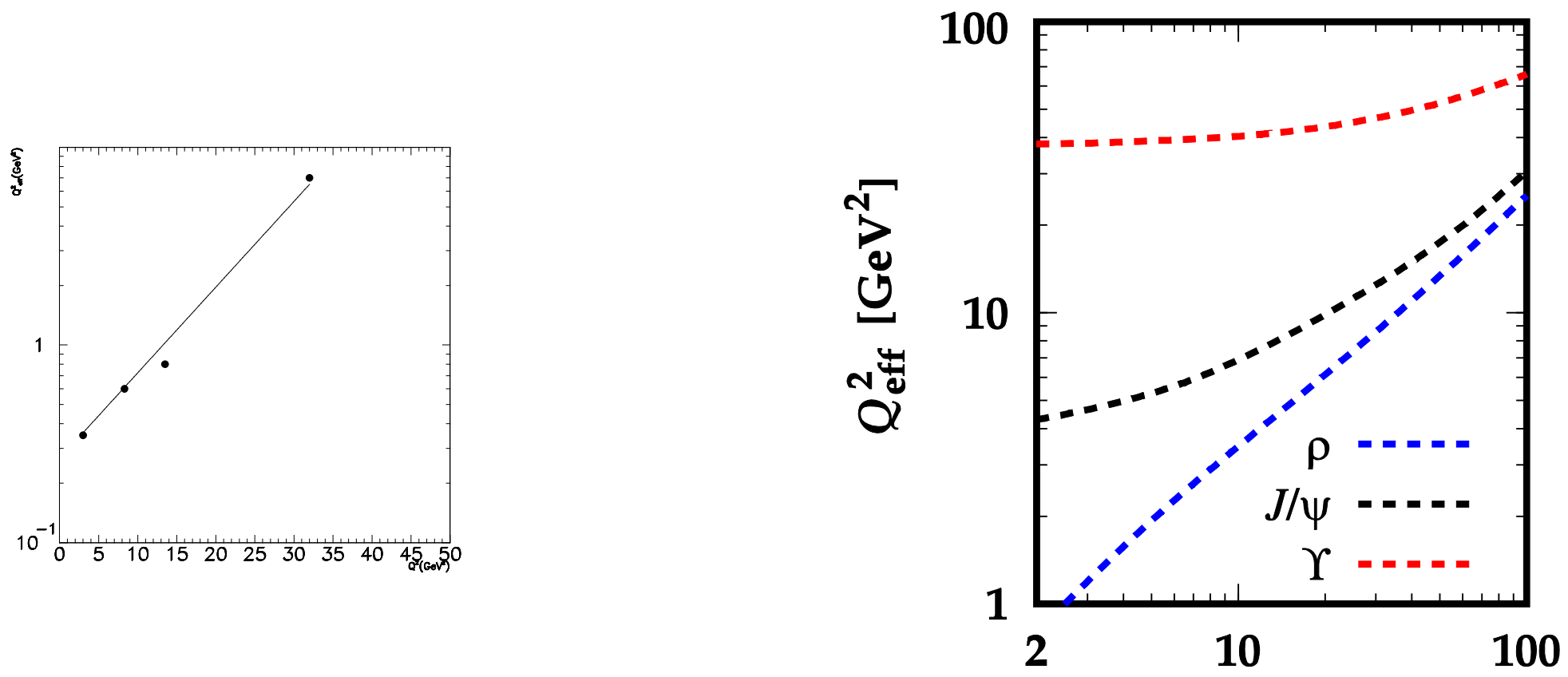


\section{Summary}

- HERA data - good source to observe interplay of soft and hard dynamics.

- Exclusive electroproduction of heavy meson - source to study PQCD.

- Need to understand issue of $Q^{2}$ eff.

Need high precision measurements of exclusive electroproduction of VM. 